\title{
Current Distribution in Helical Solenoids
}

\section{Peter F. Ryff}

Ryerson University

Ryff, P. F. (1972). Current distribution in helical solenoids. IEEE Transactions on Industry Applications, IA-8(4), 485-490.

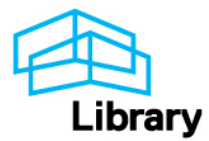




\title{
Current Distribution in Helical Solenoids
}

\author{
PETER F. RYFF, MEMBER, IEEE
}

\begin{abstract}
A method is presented for calculating the current distribution and power loss in the individual turns of helical solenoids of nonmagnetic materials. Examples are presented pertaining to 5and 14-turn coils with or without a circular billet located inside the solenoid. In addition, experimental results are included showing the excellent agreement between theory and practice.
\end{abstract}

\section{INTRODUCTION}

$\mathbf{I}^{\mathrm{N}}$ THE DESIGN of solenoids, especially those found in induction heating work, it is critically important to know the power losses on a per-turn basis in order to design the cooling system. Up to the present time, nowhere in the vast literature has there been a method for accurately predicting the current distribution and hence the power loss in the individual turns of finite-length solenoids. In most cases the total current is assumed to be distributed evenly throughout a section on the inside of the coil which has a cross section in which the depth of the turn is taken as the penetration depth $\delta$, and a width equal to that of the conductor. In some instances the deviation between measured and calculated power loss, particularly in the end turns of the solenoid, is as much as 100 percent. Another method is to approximate the coil by an array of parallel bars [1], [2]. However, serious errors are again introduced since the method does not account properly for the proximity effect caused by the curvature of the conductor in a solenoid.

Therefore, the project was undertaken which resulted in the development of the method presented here. It is now possible to calculate the current density at all points in the conductor forming the solenoid. Knowing these currents, it is relatively easy to determine the power loss per turn, the voltage drop, the ac to dc resistance ratio, and the power factor. This is important because the power loss on a turn-to-turn basis is very difficult to measure, even on experimental coils. The method is extended to include the effect on the various distributions in the coil by locating a billet inside the solenoid.

\section{System Equation}

In approaching the problem, the first consideration was how to account for the pitch of a helical coil in the calculations. Since there are no analytical solutions available to determine the magnetic field around the conductors of finite-length solenoids, an approximation was sought which would yield accurate results from an engineering

Paper TOD-71-30, approved by the Industrial Control Committee of the IEEE Industry Applications Society for publication in this Transactions. Manuscript released for publication October 25, 1971.

The author is with the Department of Electrical Technology, Ryerson Polytechnic Institute, Toronto, Ont., Canada. point of view. As has been shown [3], the magnetic field on the axis of a coil is identical to that produced by a set of parallel rings. The number of rings correspond to the number of turns and have the same cross-sectional area as that of the conductor forming the solenoid. They are placed apart at a distance which equals the pitch of the coil. This approximation does not imply that the magnetic field at the location of the conductors in a coil is identical to that created by these rings, because the rings, strictly speaking, do not properly account for the pitch angle of the solenoid. However, because of the small pitch angles encountered in most heavy-current solenoids, it appeared reasonable to assume that this approximation is valid. This assumption seems particularly appropriate when the coil is loaded.

Approximating the solenoid in this fashion, there is a method for subdividing each individual turn into a larger number of circular subconductors [4], [5]. The elements so formed are in parallel for each turn and are independent of each other, except for the requirement that the sum of all currents equals the total current in the solenoid (ring). The total currents in the rings are, of course, equal, since the current in every turn of a coil is the same. The calculations obtained using this procedure indicate that it yields very good results. This has been substantiated by extensive experimental measurements on various corresponding solenoids.

On this basis the system equation can now be derived. Starting from Maxwell's equations

$$
\begin{aligned}
& \operatorname{curl} H=J \\
& \operatorname{curl} E=-\frac{\partial B}{\partial t}=-i \omega B
\end{aligned}
$$

introducing the vector potential

$$
\operatorname{curl} \boldsymbol{A}=\boldsymbol{B}
$$

into both equations, and assuming a homogeneous space and $\operatorname{div} \boldsymbol{A}=0$, then

$$
\begin{gathered}
\nabla^{2} A=-\mu_{0} J \\
\operatorname{curl} E=-i \omega \operatorname{curl} A
\end{gathered}
$$

where $\mu_{0}\left(=4 \pi \times 10^{-7}\right.$; MKS units are used $)$ is the permeability of free space.

The general solution of (3a) is

$$
\boldsymbol{A}=\frac{\mu_{0}}{4 \pi} \int_{v} \frac{\boldsymbol{J}}{r} d v
$$

and the application of Stoke's theorem to (3b) after integration yields

$$
\oint E d l=-i \omega \oint A d l .
$$


Since $J=\sigma E$, the electric field strength can be replaced by the quotient of current density and electrical conductivity. Introducing an externally applied voltage $V_{0}$,

$$
\oint \frac{J}{\sigma} d l+i \omega \oint \boldsymbol{A} d l=V_{0}
$$

The integration in this equation is formed along a stream line. Substitution of (4a) in (5) results in

$$
\int_{l} \frac{J}{\sigma} d l+i \omega \int_{l} \frac{\mu_{0}}{4 \pi} \int_{v} \frac{J}{r} d v d l=V_{0}
$$

Assuming the current density $J$ constant along the path of integration, the volume integral may be split into an area and a line integral to give

$$
\int_{l} \frac{J}{\sigma} d l+i \omega \int_{a} J \frac{\mu_{0}}{4 \pi} \int_{l} \int_{l^{\prime}} \frac{d l d l^{\prime}}{r} d a=V_{0} .
$$

This division is possible when rotational symmetry exists. The double line integral is Neumann's integral, representing the mutual inductance

$$
L_{i, j}=\frac{\mu_{0}}{4 \pi} \int_{l} \int_{l^{\prime}} \frac{d l d l^{\prime}}{r}
$$

between the elements $i$ and $j$. In case the double integral extends over the same path, the self-inductance $L_{i i}$ is obtained. These inductances may be determined using the formulas in [6], [7]. Introducing $L_{i, j},(7)$ reduces to

$$
\frac{J_{i} l_{i}}{\sigma}+i \omega \int_{a_{j}} J_{j} L_{i, j} d a_{j}=V_{0} .
$$

Integrating the remaining integral and applying this equation to the many circular subconductors created when the turn cross sections were divided, and numbering these elements 1 to $n$, the expression becomes

$$
J_{i} l_{i}+i \omega \sigma \sum_{j=1}^{n} J_{j} L_{i, j} A_{j}=\sigma V_{0}, \quad i=1, \cdots, n .
$$

This equation represents $n$ simultaneous linear equations with complex coefficients. The solution is obtained by solving the following matrix equation, which results from (9) by expanding the terms under the summation sign and rearranging:

$$
\begin{array}{r}
{\left[\begin{array}{ccc}
\left(L_{n} A_{1}-i l_{1} / \omega \sigma\right) & \cdots & L_{1 n} A_{n} \\
\vdots & \ddots & \vdots \\
L_{n 1} A_{1} & \cdots & \left(L_{n n} A_{n}-i l_{n} / \omega \sigma\right)
\end{array}\right]\left[\begin{array}{c}
J_{1} \\
\vdots \\
J_{n}
\end{array}\right]} \\
=\left[\begin{array}{c}
-i V_{0} / \omega \\
\vdots \\
-i V_{0} / \omega
\end{array}\right] .
\end{array}
$$

\section{Voltage Distribution}

In the derivation of the system equation, the externally applied voltage $V_{0}$, or the voltage drop per turn, was taken as a constant for all the turns of the solenoid. This is not the case in any finite-length coil because the field strength decreases toward the end turns, due to the leakage fluxes, and diminishes rapidly outside the coil. In order to account for the difference in voltage drop per turn, the following procedure was applied. Using the approximations already

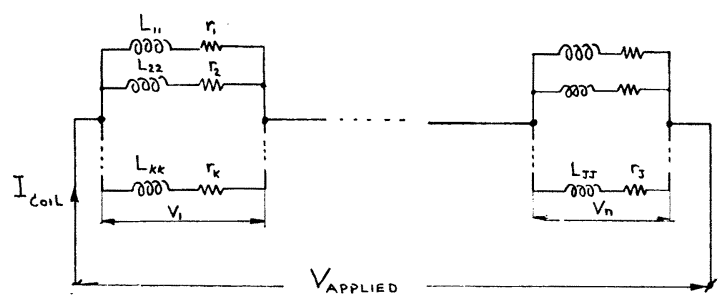

Fig. 1. Circuit representation of $n$-turn coil. Each turn is divided into $k=i / n$ sections, where $j$ is the total number of sections in all turns. All inductive elements are mutually coupled.

described and dividing the area of each turn into, say, $k$ sections, the coil may be represented by the circuit illustrated in Fig. 1.

The resistances $r_{i}, i=1, \cdots, j$, denote the corresponding dc resistance of element $i$ with a cross section of $A_{i}$. The reactance of each inductive element can be determined from

$$
\Phi_{i}=\sum_{i} L_{i, j} i_{j} \quad X_{i}=\omega L_{i}
$$

where $\omega=2 \pi \times$ frequency. Hence the impedance of each individual branch $(R L)$ is

$$
Z_{i}=r_{i}+i \omega \frac{\sum_{i} L_{i, j} i_{j}}{i_{i}} .
$$

(The use of $i$ as an index and also for the imaginary operator should be noted. They are, however, readily distinguishable.) If $k=j / n$, then the impedance of each turn is

$$
Z_{\text {turn }}=\frac{1}{\sum_{k} 1 / Z_{k}}
$$

for each parallel branch concerned. Knowing the impedances, the voltages are calculated from $V=I Z$, where $I$ is the current in the solenoid and is the same for all turns.

If the turn cross sections are not subdivided, then each turn can be represented by a single $R L$ circuit and its impedance becomes

$$
Z=R+i \omega \sum_{i} L_{i, j}
$$

since the currents in this case are equal in each branch and cancel in (12). This means that the voltage distribution for the coil can be determined without solving the system equation (10) first. However, this method may be used as a check on the results or in cases where the number of steps in the iteration process, to be described later, must be kept to a minimum.

In general, the turns are divided into a larger number of circular subconductors. The voltage distribution calculation is not simple, since the currents are yet unknown and the impedance of each element depends on all other currents, as is evident from (12). Hence, in order to determine the impedances the currents are required, or vice versa. Therefore, the voltage drops per turn were initially taken as unity. The choice of this constant is arbitrary since the current in the solenoid can be normalized afterward to obtain a predetermined value. Equation (10) can now be solved for the current densities and corresponding phase 


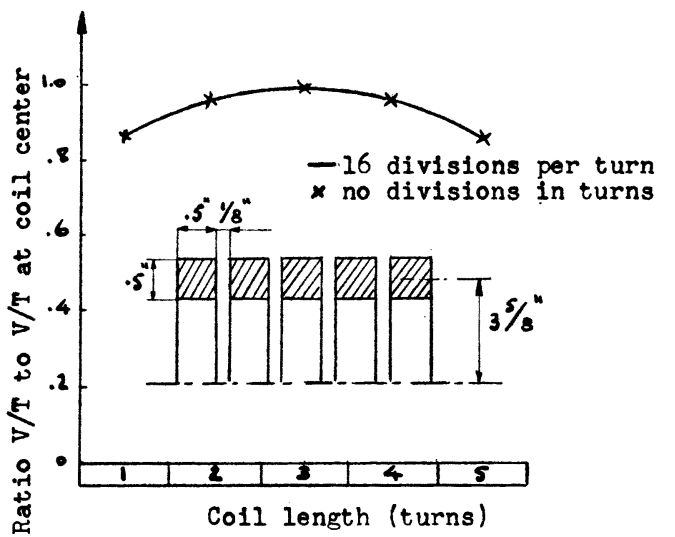

Fig. 2. Voltage distribution on 5-turn solenoid.

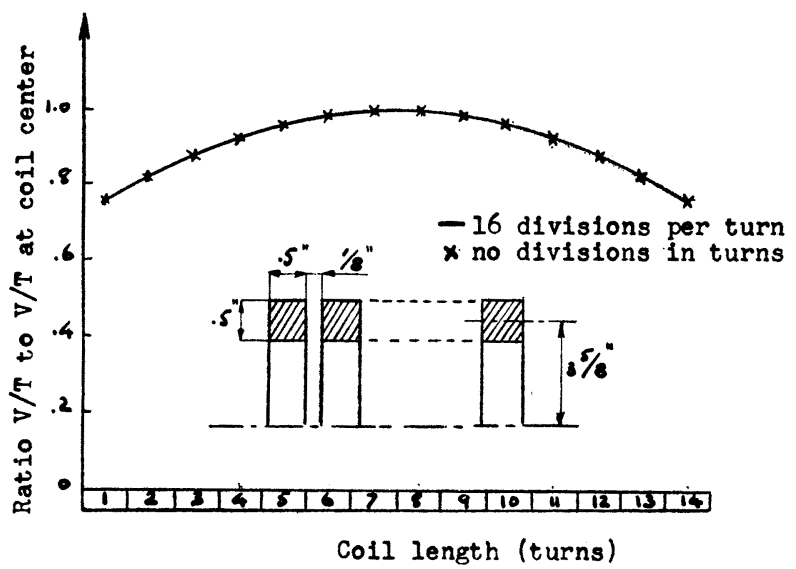

Fig. 3. Voltage distribution on 14-turn solenoid.

angle in each individual circular subconductor. The currents thus obtained are approximate, since the assumed voltage distribution across the solenoid was constant. However, the voltage distribution can now be recalculated and, using these new values, the system is solved again, yielding more accurate values.

The number of iterations required until convergence was established never exceeded four steps for any number of turns in an empty coil, while about six steps were required when loaded coils were considered. It was found that this procedure had the advantage of providing an additional check on the calculations, namely, the voltage distribution determined this way could be compared with that using the simpler method, i.e., not using subdivisions in the turns. The iteration is required since the voltages must be readjusted, which in turn modifies the currents. This modification is not linear because the volts per turn near the end of the coil are significantly less than in the midsection. This means, in effect, that the imaginary part in (12) is continually recalculated during the iteration process until convergence is established.

Some typical examples of the voltage distribution for a 5- and 14-turn solenoid are illustrated in Figs. 2 and 3, respectively. These figures also show a comparison between the voltage distribution per turn when the turn is divided into 16 circular elements and when no divisions are made.
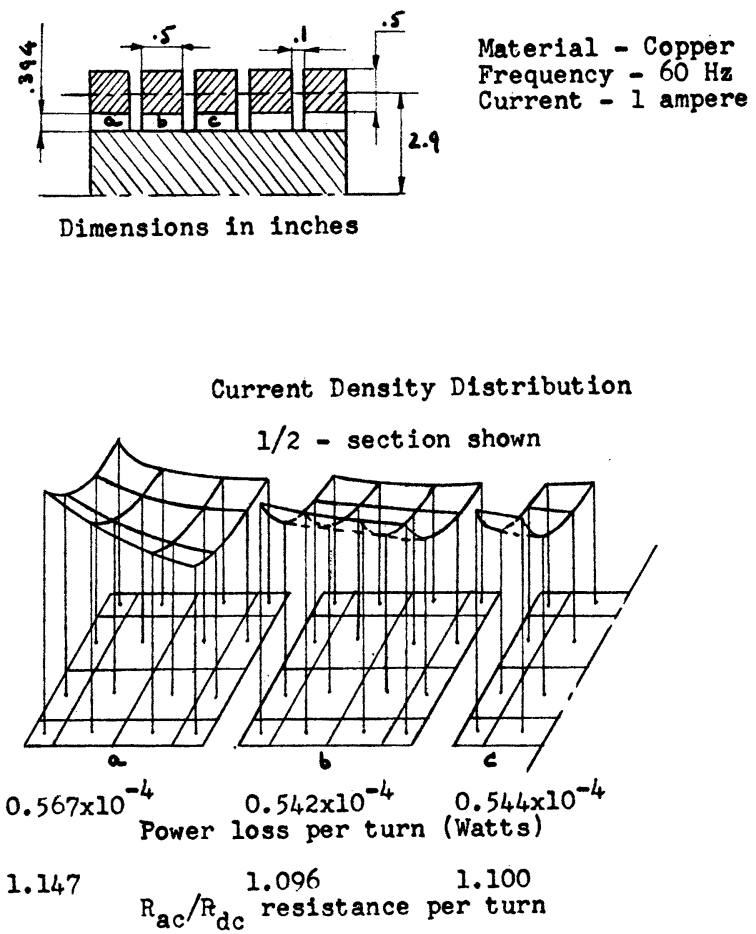

Fig. 4. Current density distribution in 5-turn solenoid with circular billet.

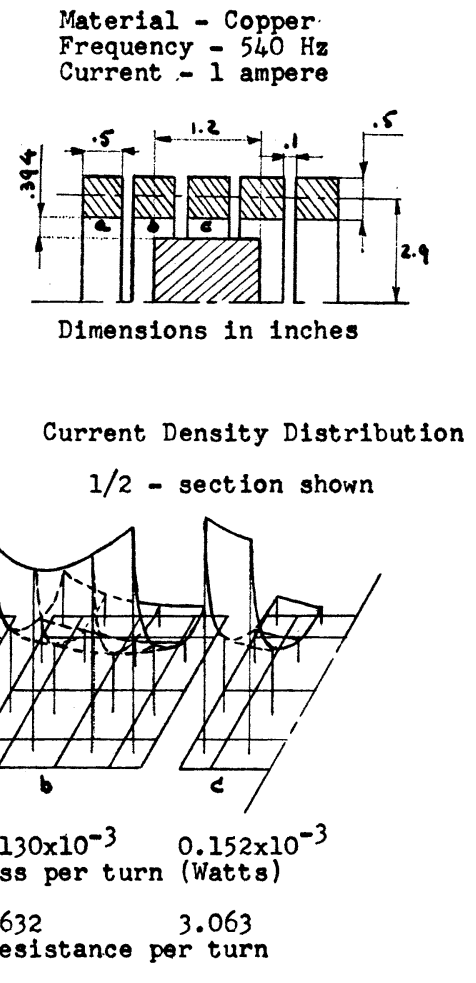

Fig. 5. Current density distribution in 5-turn solenoid with circular billet; end turns extend beyond load.

\section{Theoretical and Experimental Results}

Numerous calculations have been made in order to study the effect of the coil pitch, coil length to diameter ratio, conductor cross-sectional area, and air gap spacings between the turns. Also the effect of a circular billet, located inside the solenoid, on the current distribution in the coil has been examined. Figs. 4-8 show some of the 


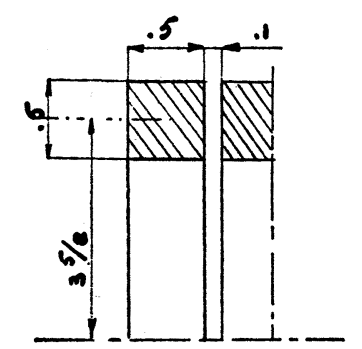

Material - Stainless Steel

Frequency - $10 \mathrm{kHz}$

Dimensions in inches

Current Density Distribution

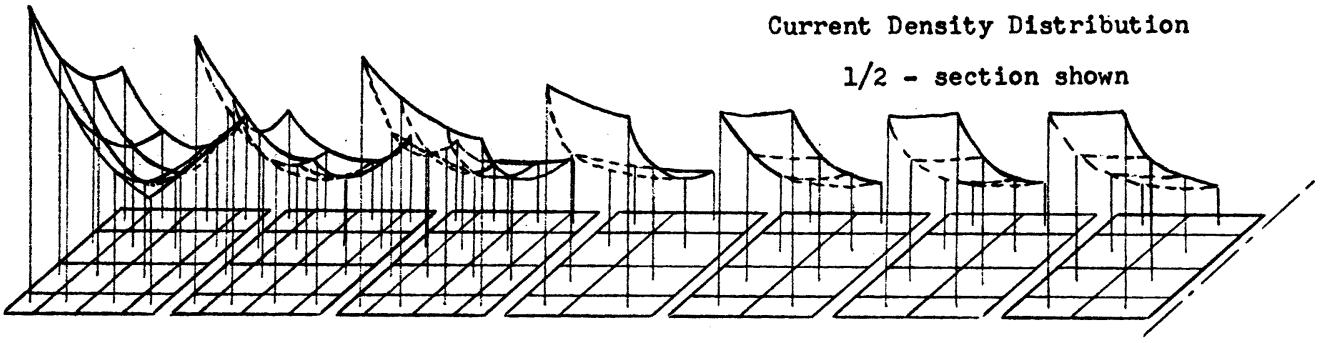

Fig. 6. Current density distribution in 14-turn solenoid.

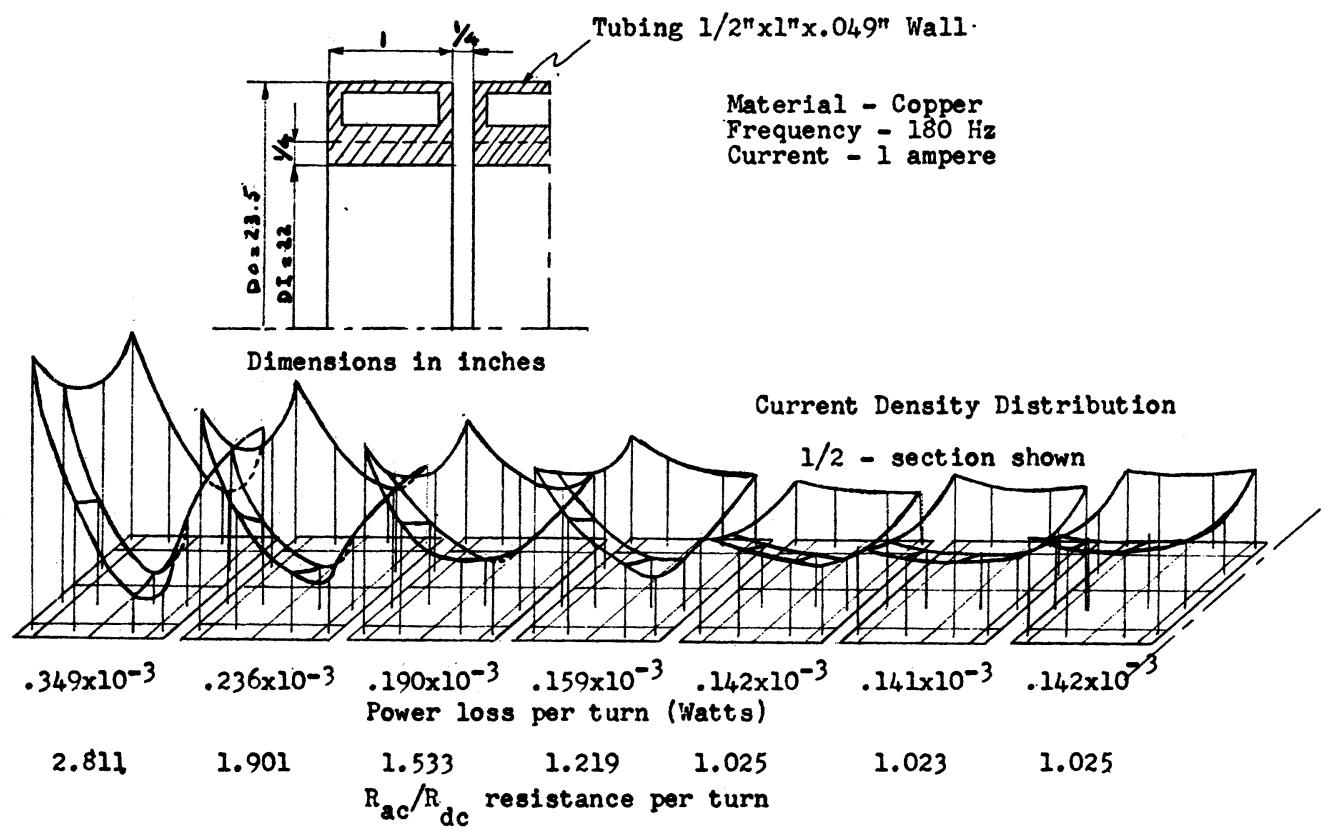

Fig. 7. Current density distribution in 14-turn hollow solenoid.

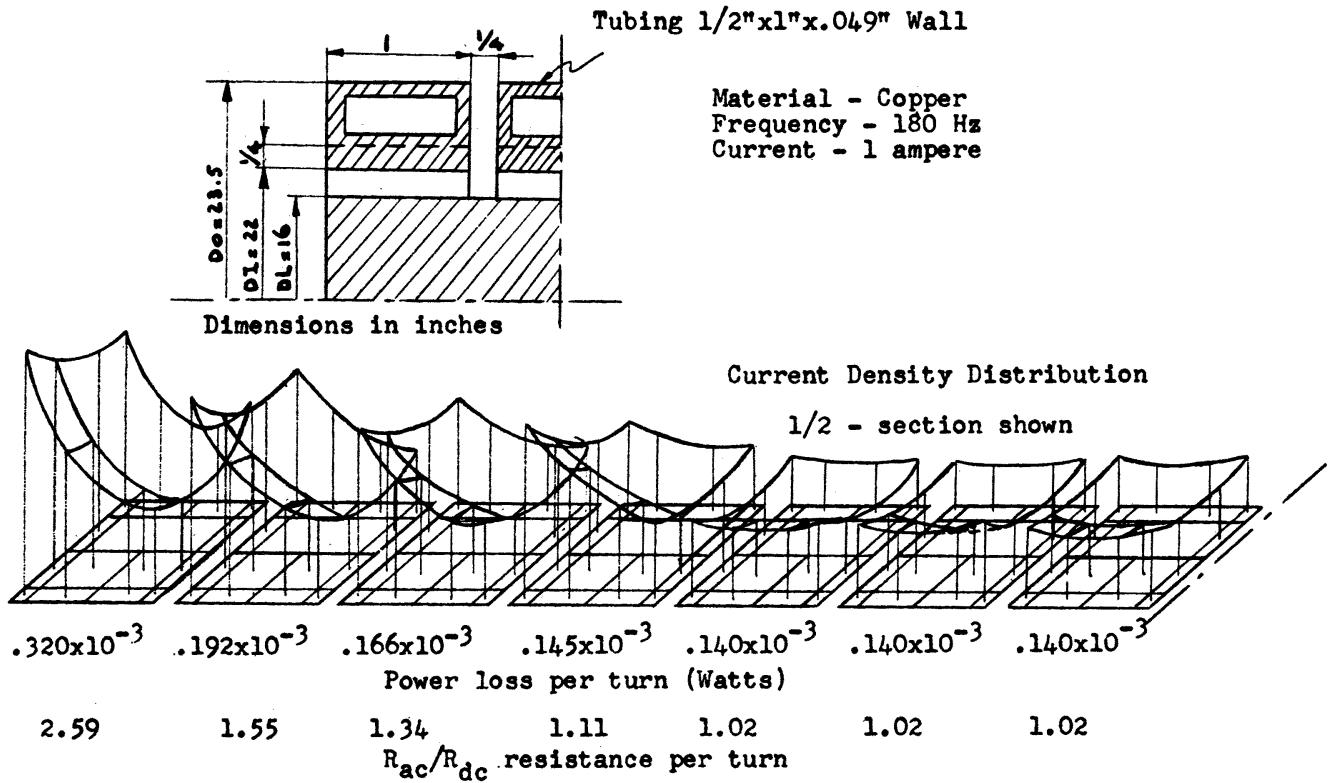

Fig. 8. Current density distribution in 14-turn hollow solenoid, including circular billet. 


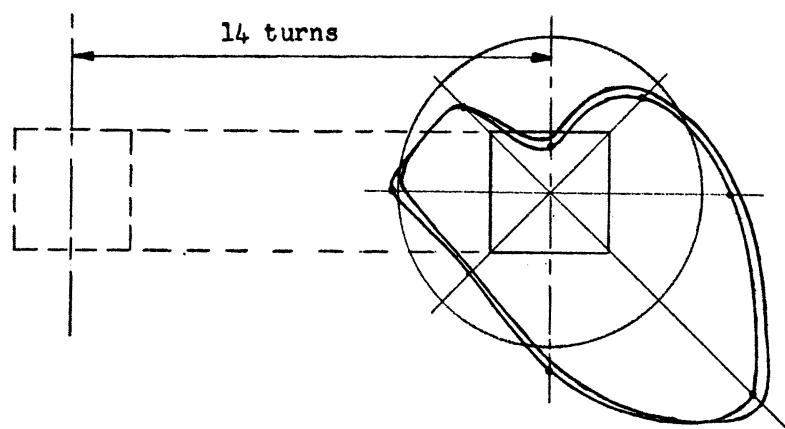

(a)

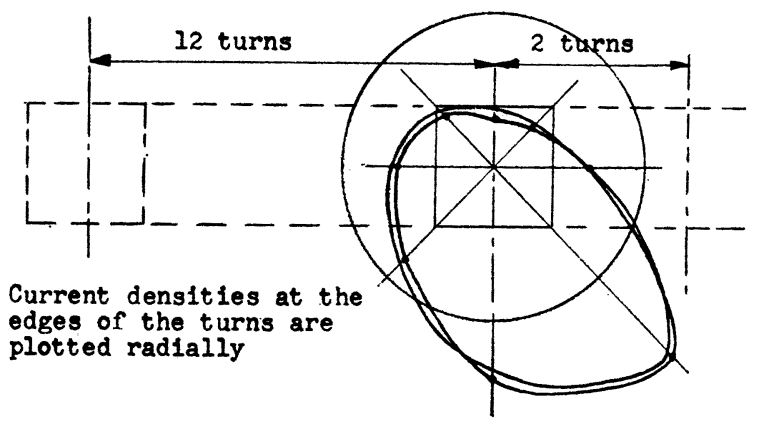

(c)

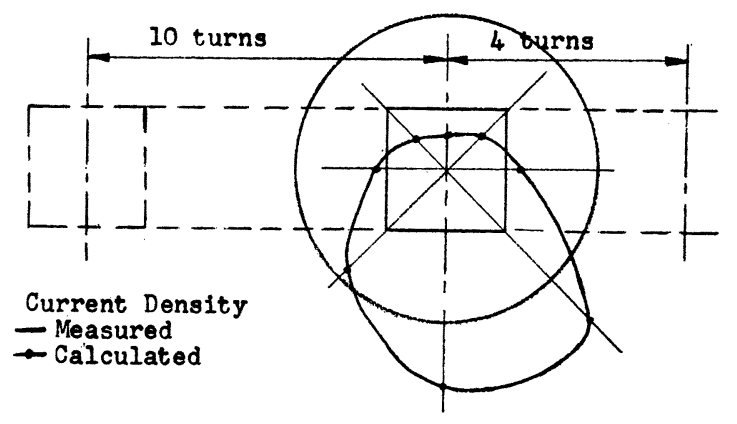

(e)

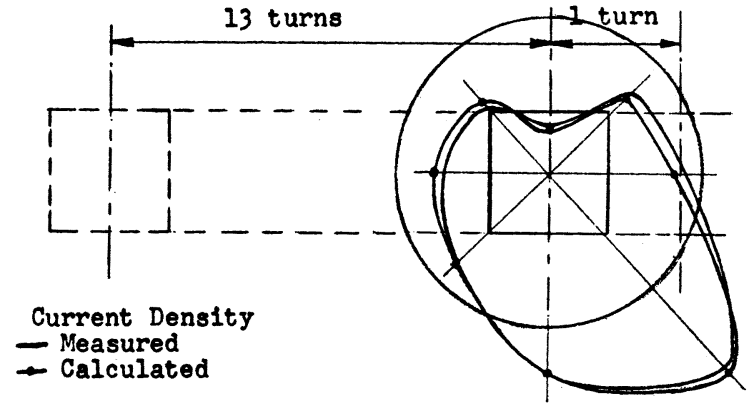

(b)

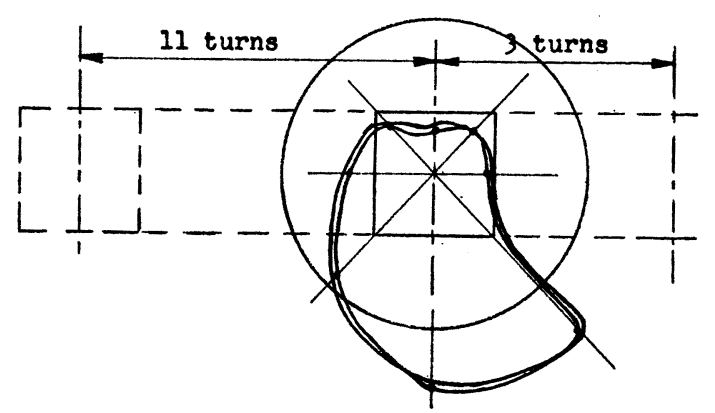

(d)

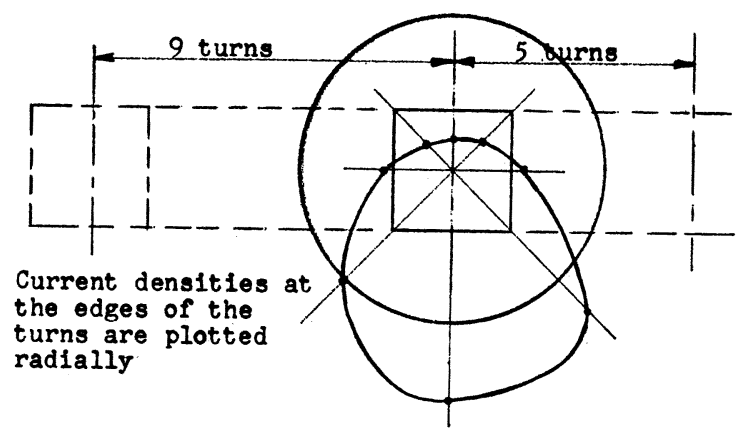

(f)

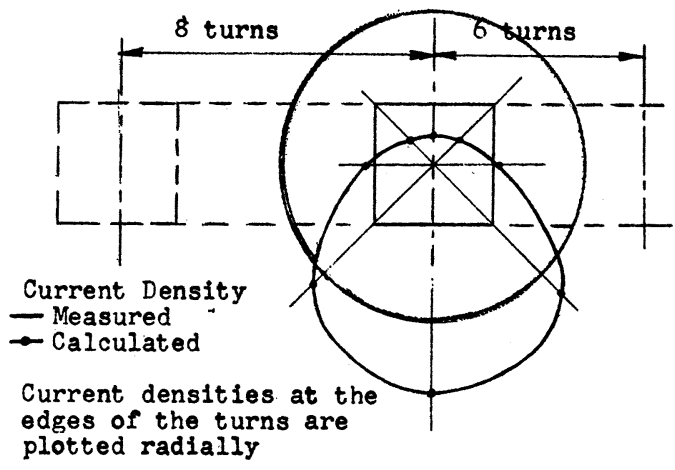

(g)

Fig. 9. Comparison of theoretical and experimental results for 14-turn stainless steel solenoid of Fig. 6 .

theoretical results in the turns of a solenoid for the particular configurations illustrated. It should be noted that in all cases each individual turn cross section has been divided into 16 circular subconductors. In addition, the power loss per turn and the ratio of alternating to direct current resistance are given. The power loss and currents possess their greatest value in the outer turns; this is as expected, since the magnetic field closes on itself around the outside of the coil. This means that the outer turns are subjected to greater cross fluxes than the inner turns, resulting in higher losses.

Fig. 9 shows a comparison of experimental and theoretical results of the coil in Fig. 6. Only the currents in the outside sections of the turns are plotted (on a radial scale) to facilitate comparison. Finally, Figs. 10 and 11 show the experimental and theoretical results of the hollow copper solenoid of Figs. 7 and 8. It is easy to see the effect of the load on the power loss distribution, namely, it tends to 


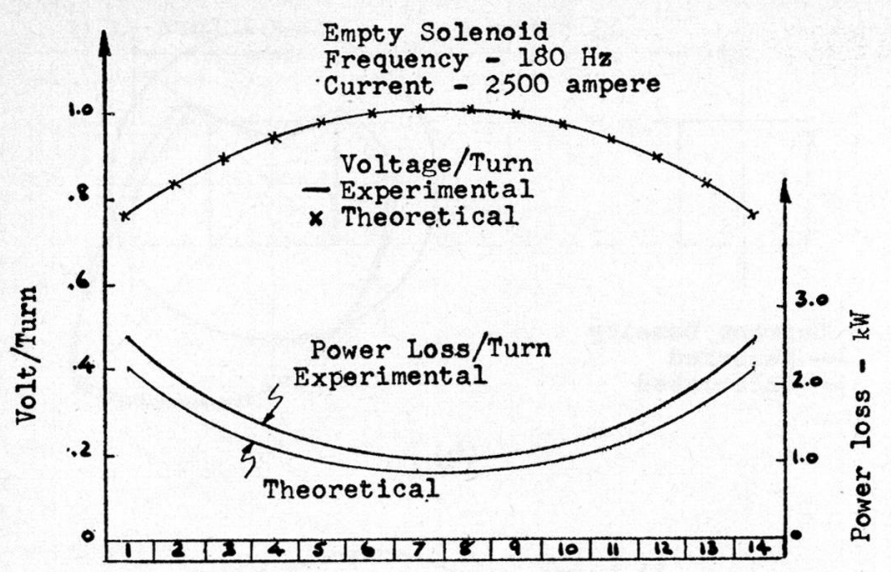

Fig. 10. Comparison of theoretical and experimental results for solenoid in Fig. 7.

"straighten" it out. The experimental power loss distribution is somewhat above the theoretically predicted values. This is due to an iron yoke being present in the experimental setup, which we know from prior work will increase the power loss.

\section{Conclusions}

The calculated results have been compared with experimentally obtained data for numerous examples and the agreement in all cases was excellent from an engineering point of view. Therefore, with the method presented it is now possible to analyze a solenoid and obtain a good assessment of a coil prior to construction.

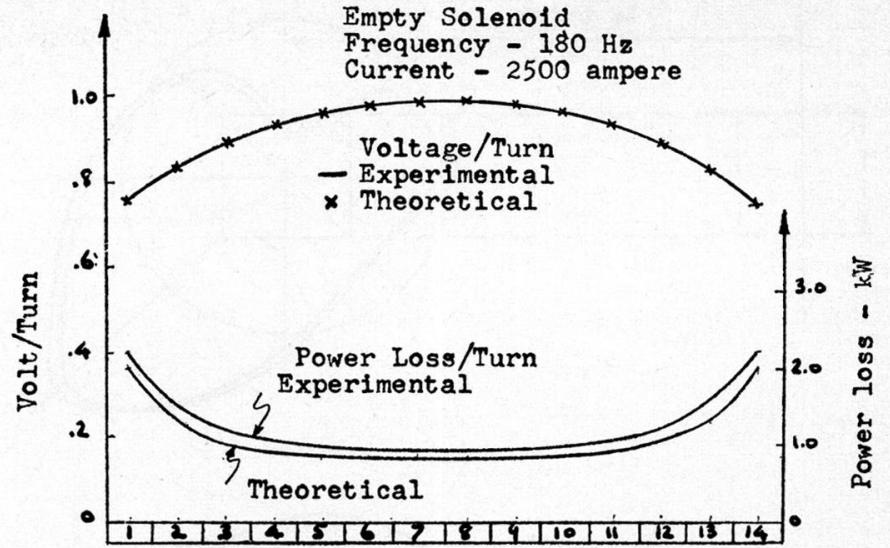

Fig. 11. Comparison of theoretical and experimental results for solenoid in Fig. 8.

\section{REFERENCES}

[1] S. Butterworth, "Eddy current losses in cylindrical conductors with special application to the alternating current resistance of short coils," Phil. Trans. Roy. Soc. London, p. A222, 1921.

[2] S. Butterworth "On the alternating current resistance of solenoidal coils," Proc. Roy. Soc., Ser. A, pp. A1-7, 1925.

[3] H. Buchholtz, Potentialfelder. Berlin: Springer, 1957.

[4] P. P. Biringer, P. F. Ryff, and R. S. Segsworth, "Current distribution in conductors of arbitrary cross section considering corner effects," in Conf. Rec., 1968 IEEE IGA Group Annu. Meeting, pp. 105-116.

[5] P. F. Ryff, P. P. Biringer, and P. E. Burke, "Calculation methods for current distribution in single-turn coils of arbitrary cross section," IEEE Trans. Power App. Syst., vol. PAS-89, pp. 228-232, Feb. 1970.

[6] F. W. Grover, Inductance Calculations, Working Formulas and Tables. New York, Dover: 1945.

[7] G. R. Olshausen, "Absolute formulas for the mutual inductance of coaxial solenoids," Phys. Rev., Dec. 1910.

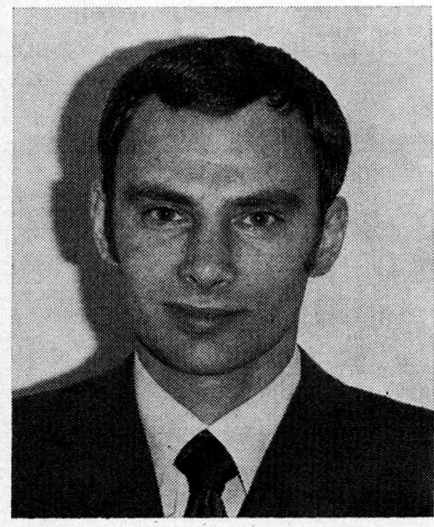

Peter F. Ryff (S'66-M'66) was born in Amsterdam, The Netherlands, on August 27, 1935. He graduated from the Engineering College of Amsterdam, Amsterdam, in 1958 and received the B.A.Sc., M.A.Sc., and Ph.D. degrees in electrical engineering from the University of Toronto, Toronto, Ont., Canada, in 1966, 1967, and 1969, respectively.

From 1958 to 1964 he was employed by Honeywell, Inc., Toronto, and became their Field Engineering Representative. From 1969 to 1970 he was employed by General Time of Canada, Ltd., Peterborough, Ont., as a Research Engineer in the field of electromagnetic and solidstate time-base components. In 1970 he joined the Electrical Technology Department of Ryerson Polytechnical Institute, Toronto. His present research interests are in the field of induction heating. He has authored several technical publications on eddy-current loss calculations.

Dr. Ryff is a member of the Association of Professional Engineers of the Province of Ontario. 